\title{
Ultrasound-guided supraclavicular brachial plexus block in a patient with a cervical rib
}

\author{
Tatsunori Watanabe, MD • Kazuhito Yanabashi, MD • Koji Moriya, MD • \\ Yutaka Maki, MD, PhD • Naoto Tsubokawa, MD, PhD • Hiroshi Baba, MD, PhD
}

Received: 12 December 2014/ Accepted: 26 January 2015 / Published online: 6 February 2015

(C) Canadian Anesthesiologists' Society 2015

\section{To the Editor,}

Anatomical variants can make regional anesthesia challenging even when ultrasound guidance is employed. This report describes our experience while performing ultrasound-guided supraclavicular brachial plexus block in a patient with a cervical rib. Patient consent was obtained for publication of this report.

A 57-yr-old male (height, $160 \mathrm{~cm}$; weight, $44 \mathrm{~kg}$ ) was admitted with an open fracture of his right middle phalanx and a lacerated digital artery of his right index finger. His medical history included type 2 diabetes mellitus treated with oral hypoglycemic drugs. The preoperative chest radiograph showed a left cervical rib (Figure A) and a normal right clavicle. He reported no symptoms of thoracic outlet syndrome in either of his upper limbs. An open reduction and internal fixation of the middle phalanx and anastomosis of the digital artery were performed.

The nerves surrounding the subclavian artery were identified by placing a high-frequency $(6-15 \mathrm{MHz})$ ultrasound probe $\left(\mathrm{EDGE}^{\circledR} ;\right.$ SonoSite, Tokyo, Japan) above the clavicle. A bony structure was identified lateral to the nerves, indicated by a smooth hyperechoic edge with a posterior acoustic shadow (Figure C). In light of this finding, we reassessed the chest radiographs and magnified the right cervical area where a right cervical rib was confirmed (Figure B). While the cervical rib partially

T. Watanabe, MD $(\bowtie) \cdot H$. Baba, MD, PhD

Division of Anesthesiology, Niigata University Graduate School

of Medical and Dental Sciences, Niigata, Japan

e-mail: tatsu-w@med.niigata-u.ac.jp

T. Watanabe, MD · K. Yanabashi, MD - K. Moriya, MD .

Y. Maki, MD, PhD - N. Tsubokawa, MD, PhD

Niigata Hand Surgery Foundation, Niigata, Japan obstructed the needle path for a lateral in-plane, supraclavicular approach to the brachial plexus, we were able to inject $10 \mathrm{~mL}$ of both $1 \%$ mepivacaine and $0.5 \%$ bupivacaine (total $20 \mathrm{~mL}$ ) with ultrasound guidance. The distribution and duration of the sensory block were sufficient for a surgical repair lasting $109 \mathrm{~min}$. Postoperative neuropathy was not observed.

Cervical ribs are supernumerary ribs which arise from the seventh cervical vertebra and are present in 0.05-3.0\% of the population. ${ }^{1}$ In the study by Viertel et al., the authors found that almost $60 \%$ of patients with cervical ribs had a unilateral rib, while the remaining patients had bilateral cervical ribs. ${ }^{1}$ Although the majority of cervical ribs are not clinically relevant, these anomalies may cause thoracic outlet syndrome. ${ }^{2}$ Complete cervical ribs attach to the lateral first rib through a true joint, whereas incomplete cervical ribs typically have a ligamentous extension to the first rib. $^{3}$ The structure is usually diagnosed by plain radiography or computed tomography.

Structures other than cervical ribs may also be observed in the supraclavicular area. Calcified cervical lymph nodes may appear similar to bones on ultrasound imaging. Lymph nodes may become calcified due to sarcoidosis, lymph node metastasis from malignant cancers (e.g., thyroid, lung, or breast cancer), and infectious diseases (e.g., tuberculosis). ${ }^{4}$ When a bone-like structure is observed surrounding the brachial plexus on the supraclavicular ultrasound image, detailed radiographic imaging is indicated to rule out these less benign conditions.

While a supraclavicular approach to the brachial plexus was used in this case, the cervical rib was also identified on the ultrasound image using an interscalene approach (Figure D). We prefer to use the lateral in-plane supraclavicular approach to avoid vascular injury and to visualize the entire needle path. If a larger cervical rib had 
Figure A) Preoperative chest radiograph of a 57-yr-old male. B) Magnified view of the right cervical area on the same preoperative chest radiograph. The right cervical rib can be clearly identified. C) Ultrasound image showing the

supraclavicular brachial plexus block performed in this case.

The dashed arrow indicates the needle path chosen. D)

Ultrasound image showing an alternate technique, an interscalene brachial plexus block. The dashed arrow indicates the suggested needle path. White arrow $=$ cervical rib; Dotted circle = nerve; SCA = subclavian artery; $\mathrm{IJV}=$ internal jugular vein; ASM $=$ anterior scalene muscle; $\mathrm{MSM}=$ medial scalene muscle;

$\mathrm{SCM}=$ sternocleidomastoid muscle
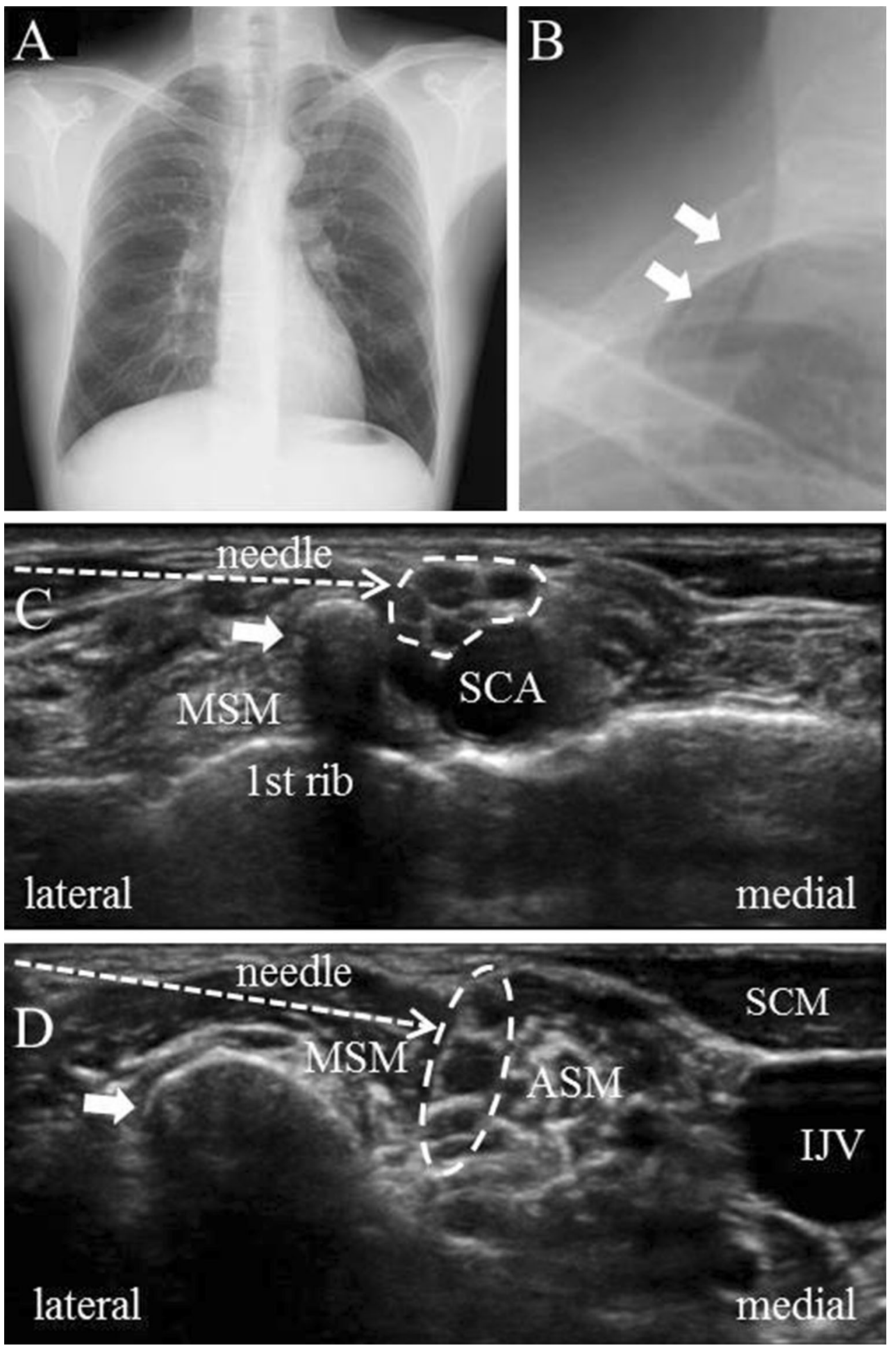

hindered the lateral approach, either an in-plane approach from the medial side or an out-of-plane approach could have been considered.

If an unusual bone-like structure is observed in the ultrasound image obtained during a supraclavicular or interscalene brachial plexus block, it is necessary to confirm the diagnosis of a cervical rib on the radiographic image and to plan the needle path accordingly.

Conflicts of interest None declared. 


\section{References}

1. Viertel VG, Intrapiromkul J, Maluf $F$, et al. Cervical ribs: a common variant overlooked in CT imaging. AJNR Am J Neuroradiol 2012; 33: 2191-4.

2. Walden MJ, Adin ME, Visagan $R$, et al. Cervical ribs: identification on MRI and clinical relevance. Clin Imaging 2013; 37: $938-41$.
3. Thompson $R W$, Emery VB. Thoracic outlet procedure. In: Kaiser LR, Kron IL, Spray TL, editors. Mastery of Cardiothoracic Surgery - Third Edition. Philadelphia: Lippincott Williams \& Wilkins; 2012: 242-52.

4. Tortorich J, Woods M, Shintaku W, Anderson KM. Diagnostic considerations of calcified lymph nodes. J Tenn Dent Assoc 2013; 93: 8-10; quiz 11-2. 